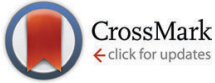

Cite this: Chem. Commun., 2016, 52,8608

Received 23rd May 2016. Accepted 8th June 2016

DOI: $10.1039 / \mathrm{c} 6 \mathrm{cc} 04312 \mathrm{~g}$

www.rsc.org/chemcomm

\title{
Stress-induced colouration and crosslinking of polymeric materials by mechanochemical formation of triphenylimidazolyl radicals $\dagger$
}

\author{
F. Verstraeten, R. Göstl* and R. P. Sijbesma*
}

\begin{abstract}
Under mechanical stress, the hexaarylbiimidazole (HABI) motif can cleave to triphenylimidazolyl radicals when incorporated into a polymer matrix. The mechanically produced coloured radicals can initiate secondary radical reactions yielding polymer networks. Thus, the HABI mechanophore combines optical reporting of bond scission and reinforcement of polymers in a single molecular moiety.
\end{abstract}

Mechanical degradation is an inconvenient yet inevitable effect of repeated deformation when using polymer-based materials. Stress-induced bond scission, a microscopic process, eventually causes macroscopic failure of the material. Recent research demonstrates impressively how generally unspecific and undesired bond scission can be altered into constructive processes. ${ }^{1-5}$ Commonly, this is achieved by incorporating mechanically activatable molecular moieties (mechanophores) into polymer architectures. Those mechanophores then either act as probes for the visualization of stress or initiate the repair of the damaged material. Amongst the motifs for stress-report, functional moieties changing their optical properties upon the application of stress are highly desirable as they allow the visualization of areas under heavy loading. ${ }^{5-7}$ Concomitantly, stress-induced repair has been realized by mechanochemically activated catalytic metathesis polymerization $^{8-10}$ or stress-induced crosslinking reactions. ${ }^{11-14}$ Consequently, the implementation of both the ability to visualize as well as repair broken bonds in a polymeric material can be seen as a significant advance in the field of materials science, as recently demonstrated by Weng, Boulatov and co-workers. ${ }^{11}$

Motifs relying on the generation of stabilized radicals are particularly attractive for this endeavour as they combine features favourable for optical detection and for the formation of new chemical bonds. While the delocalization of mechanically generated radicals into a $\pi$-system can produce strongly absorbing

Technische Universiteit Eindhoven, Molecular Science and Technology, Group Supramolecular Polymer Chemistry, P.O. Box 513, 5600 MB Eindhoven, The Netherlands. E-mail: r.gostl@tue.nl,r.p.sijbesma@tue.nl

$\dagger$ Electronic supplementary information (ESI) available: Details on synthesis and characterization as well as mechanical scission experiments. See DOI: 10.1039/ c6cc04312g chromophores, the controlled reactivity of these radicals may be used to initiate constructive secondary reactions, such as polymerizations. The former was recently demonstrated by Otsuka and co-workers relying on the reversibly induced cleavage of the diarylbibenzofuranone moiety ${ }^{15-17}$ while the latter is reported exploiting unspecific, ultrasound-induced polymer scission to unstabilized radicals in solution yielding end-capped, chainextended or grafted polymers. ${ }^{18-21}$

So far only little attention has been paid to the use of hexaarylbiimidazole in polymer mechanochemistry. This motif reversibly cleaves into highly coloured triphenylimidazolyl (TPI) radicals upon the application of light, heat or pressure (Scheme 1a). ${ }^{22-25}$ Moreover, HABI derivatives are already employed as radical photoinitiators in photoimaging systems ${ }^{26}$ as well as for the realization of de-crosslinkable polymer networks. ${ }^{27,28}$ Most importantly however, the TPI's ability to initiate radical polymerizations mediated by thiol chain transfer agents ${ }^{29}$ (Scheme 1 b) renders the implementation of HABI to deliver stress report and bond repair highly promising.
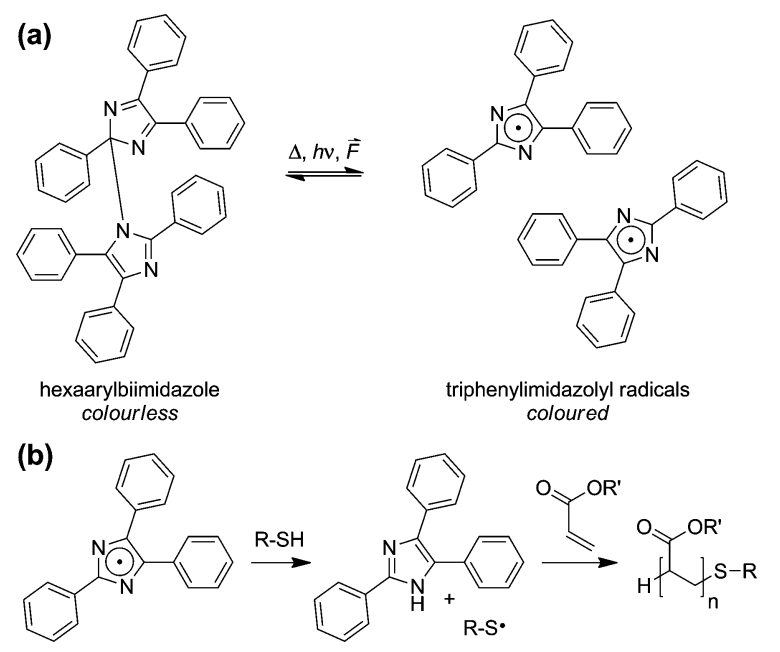

Scheme 1 (a) Reversible dissociation of HABI into TPI radicals. (b) Polymerization initiated by TPI radicals. 
Here we show the mechanical activation of this motif by transmitting force employing different polymer architectures, in solution as well as in physically crosslinked networks in the solid state, to achieve optical report over the mechanical activation and exploit the bond-scission to initiate radical reactions.

To gain insight into the mechanochemical activity of the HABI motif, linear poly(methyl acrylate) chains containing the functional moiety in their centre (PMA-1) were synthesized for ultrasonication activation in the dissolved state (Scheme 2). Alkylation of commercial 4-hydroxybenzaldehyde with 6-chlorohexanol yielded ether $\mathbf{4}$ which, in turn, reacts with benzil followed up by saponification of the phenyl acetate side-product giving imidazole 3. ${ }^{30}$ Esterification with $\alpha$-bromoisobutyryl bromide to initiator 2 and subsequent $\mathrm{Cu}(0)$-mediated living radical polymerization with methyl acrylate (SET-LRP) result in PMA-2 $\left(M_{\mathrm{n}}=98 \mathrm{kDa}\right.$, $Ð=1.06) .{ }^{31}$ Oxidative coupling to PMA-1 was carried out with $\mathrm{PbO}_{2}$ after the polymerization step instead of before because we found that the dynamic equilibrium of HABI with its TPI radicals is incompatible with the conditions of most living radical polymerizations. $^{32}$ Relatively high molecular weights for PMA-2 were chosen as we discovered that increasing the molecular weight improved the coupling efficiency. Due to the HABI's dynamic character and the relatively high dilution of the functional groups, conversion to the coupled product was generally incomplete and roughly 50\% of PMA-2 remained in the final product.

For experiments in the solid state physically crosslinked polyurethane PU-1 was prepared by oxidation of imidazole 3 with $\mathrm{K}_{3} \mathrm{Fe}(\mathrm{CN})_{6}$ to give diol 1, which then yielded the final product in a polycondensation reaction with hexamethylene diisocyanate and poly(tetrahydrofuran) $\left(M_{\mathrm{n}}=2 \mathrm{kDa}\right)$ using a procedure employed by us in an earlier work. ${ }^{33}$

Mechanochemical activation in solution was carried out by ultrasonication of PMA-1 in benzene. A first indication that the desired cleaving process is occurring at the mechanophore can be gathered by monitoring the gel permeation chromatography (GPC) refractive index (RI) detector traces (Fig. 1a). The high molecular weight peak situated at around $200 \mathrm{kDa}$ decreases rapidly and adds to the original low molecular weight peak of PMA-2 at $100 \mathrm{kDa}$. We attribute the peak-shoulder arising at around $50 \mathrm{kDa}$ to unspecific secondary chain-scission due to the large contour length of PMA-2. ${ }^{1} \mathrm{H}-\mathrm{NMR}$ measurements clearly confirm that scission occurs mostly at the HABI-moiety (Fig. 1b) by revealing the loss of the characteristic protons at 7.8 and $8.0 \mathrm{ppm}$ leaving only the TPI signals of PMA-2. Additionally, thermal activation was ruled out by keeping a benzene solution of PMA-1 at room temperature without applying ultrasound irradiation proving that thermal dissociation to the TPI radicals cannot be the origin of the observed cleaving process as the HABI's characteristic signals can still be retrieved. To explore the mechanochemical origin of the sonochemical activation of PMA-1 and to further rule out thermal effects small molecule $\mathbf{1}$ was subjected to similar sonication conditions as PMA-1 (Fig. 1c). Since the limiting molecular weight for mechanochemical activation is typically around 30-40 kDa, we expected this low-molecular weight mechanophore not to show mechanoresponsive behaviour. ${ }^{34,35}$ Indeed, from the ${ }^{1} \mathrm{H}-\mathrm{NMR}$ spectra it becomes clear that neither thermal dissociation nor irradiation with ultrasound of 1 lead to the formation of TPI or other considerable degradation of the HABI-moiety during the monitored time span.

Having confirmed that the HABI-motif can be employed as potent mechanophore we then advanced our system to the solid state. Initial solid state activation experiments on PU-1 were carried out by compression with a pellet press and a blue coloration of the compressed area indicating formation of the TPI radicals can be observed (Fig. S4, ESI $\dagger$ ). For reference purposes a similar polyurethane with solution-blended instead

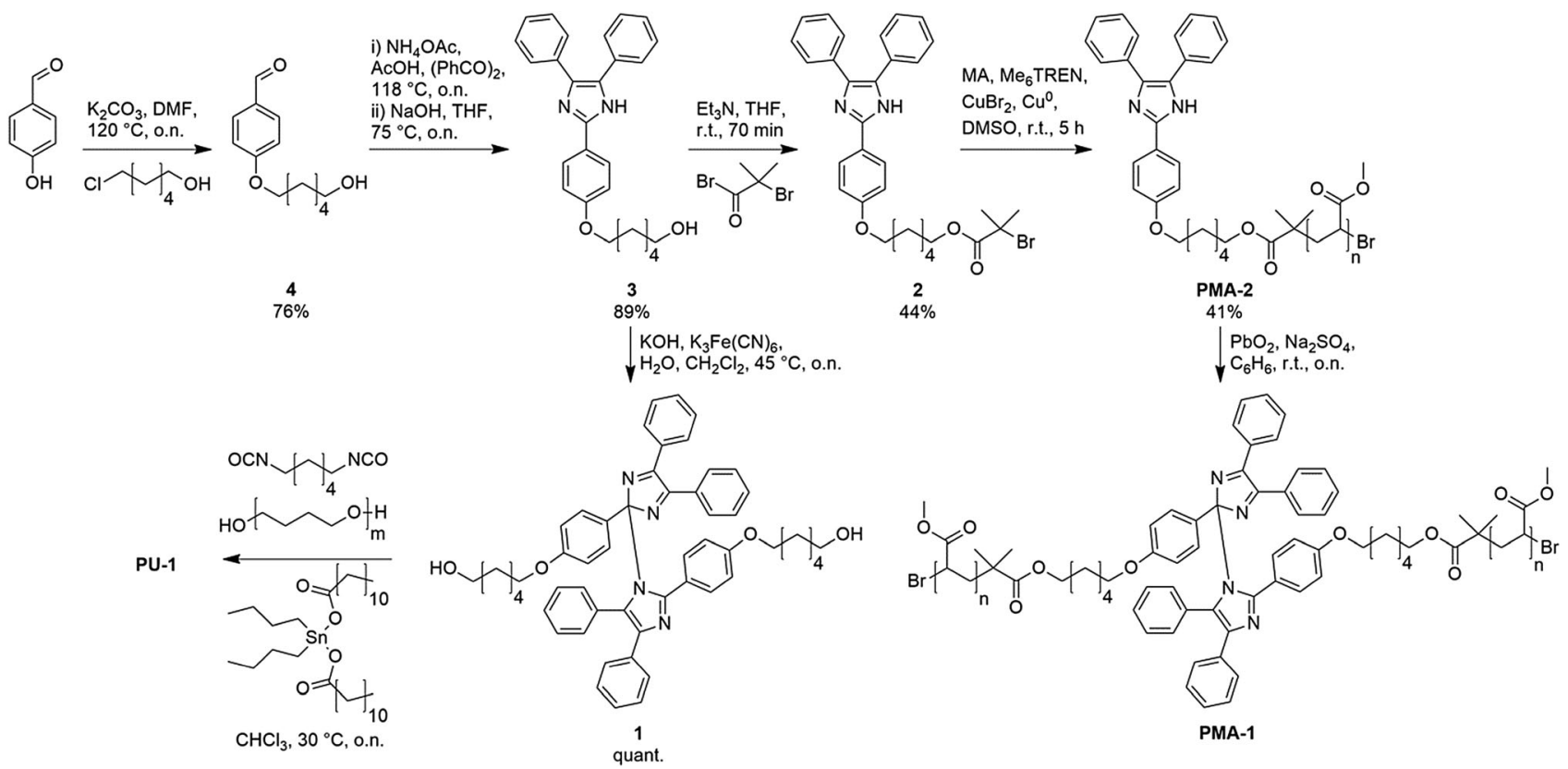

Scheme 2 Synthesis of $\mathrm{HABI}$-containing target polymers PMA-1 and PU-1. 

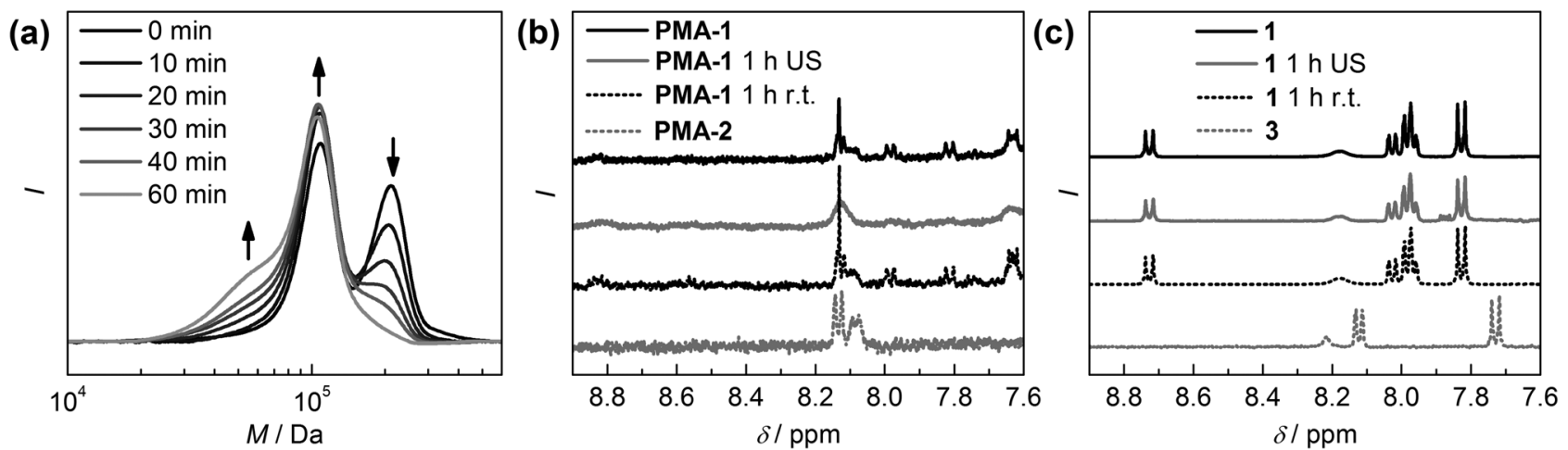

Fig. 1 Activation of PMA-1 by ultrasonication in $\mathrm{C}_{6} \mathrm{H}_{6}$. (a) GPC RI traces over the course of the sonication experiment. (b) ${ }^{1} \mathrm{H}-\mathrm{NMR}$ spectra in $\mathrm{C}_{6} \mathrm{D}_{6}$ of PMA-1, PMA-1 after $1 \mathrm{~h}$ of sonication, PMA-1 after $1 \mathrm{~h}$ at r.t. and PMA-2. (c) ${ }^{1} \mathrm{H}-\mathrm{NMR}$ spectra in $\mathrm{C}_{6} \mathrm{D}_{6}$ of small molecule 1,1 after $1 \mathrm{~h}$ of sonication, 1 after $1 \mathrm{~h}$ at r.t. and 3 .

of covalently attached HABI was prepared and also gave a slight coloration during compression. Even though the magnitude of activation appears to be far less in the reference experiment, thermo- or piezochromic effects ${ }^{24,25}$ during compression could hence not completely be ruled out as a source of TPI radical generation.

Inspired by Otsuka and co-workers ${ }^{16}$ we eliminated these effects by rapid freezing of solvent-swollen networks to mechanochemically activate PU-1. Freezing constrains the polymer chains in their translational motion rendering rate of macromolecule migration considerably lower than the rate of solvent migration. Consequently the solvent crystallizes thus straining the polymer chains. ${ }^{36}$ For this purpose PU-1 as well as polyurethane containing solution-blended HABI were first swollen in DMF and then immersed into liquid $\mathrm{N}_{2}$. From Fig. $2 \mathrm{a}$ and $\mathrm{c}$ it becomes clear that the reference polyurethane with HABI not covalently attached to the polymer backbone does not change its colour upon freezing and thus does not cleave the HABI-moiety through the application of mechanical stress. However, Fig. $2 \mathrm{~b}$ and d clearly indicate the formation of coloured TPI radicals after freezing of PU-1 unequivocally confirming the mechanochemical origin of the observed activation. Despite the fast radical recombination of TPI radicals to form HABI in systems with limited translational diffusion, we could achieve stress-sensing with a weightfraction of only $4 \% 1$ which is a considerable improvement compared to the radical systems reported in the literature ${ }^{15,16}$ (a movie of the fading coloration of compressed PU-1 in mpg format is available in the ESI $\dagger$ ).

As freezing of the swollen gel yields the most unambiguous results, proof of concept that a radical reaction can be induced

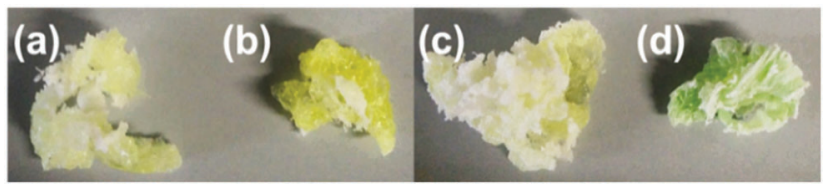

Fig. 2 DMF-swollen samples of (a) reference polyurethane and (b) PU-1 before freezing. (c) DMF-swollen reference polyurethane and (d) PU-1 after freezing in liquid $\mathrm{N}_{2}$. mechanochemically was provided by freezing PU-1 swollen with a mixture of ethylene glycol dimethacrylate (EGDMA) and $50 \mathrm{~mol} \%$ methyl 3-mercaptopropionate (MMP). Once the TPI radicals are generated, hydrogen abstraction of the thiol occurs which then initiates the polymerization to a poly(EGDMA) network (compare Scheme 1b) ${ }^{29}$ Note that comparatively high amounts of thiol were needed to achieve a sufficient amount of growing chains as the initiation is mostly limited by diffusion of the thawing MMP to the TPI moieties. A first indication that this cascade is initiated by mechanical stress was gathered by comparing the colours of the frozen samples of PU-1 swollen in EGDMA in the presence or absence of MMP upon thawing (Fig. S5a, ESI $\dagger$ ). While a reference sample of PU-1 swollen in EGDMA turns brightly green, PU-1 swollen in EGDMA with $50 \mathrm{~mol} \%$ MMP decolourizes rapidly suggesting that TPI radicals are formed in the latter system but quickly abstract a hydrogen atom from MMP. The consecutive initiation of crosslinking by MMP radicals is strongly indicated by the formation of a DMF-insoluble white residue after 3 successive freezing and thawing cycles in PU-1 swollen with both MMP and EGDMA (Fig. S5b, ESI $\dagger$ ), but not in PU-1 swollen with EGDMA alone.

FTIR-spectroscopy of this residue confirms the absence of the signals indicative for PU-1 suggesting complete dissolution of the polyurethane (Fig. 3a and b). However, the typical $\mathrm{C}=\mathrm{O}$ stretch $\left(1719 \mathrm{~cm}^{-1}\right)$ and $\mathrm{C}=\mathrm{C}$ stretch $\left(1638 \mathrm{~cm}^{-1}\right)$ absorptions of EGDMA can be retrieved in the solid residue pointing towards the formation of a poly(EGDMA) network. Moreover, the successful polymerization of EGDMA is indicated by the decreasing intensity of the $\mathrm{C}=\mathrm{C}$ stretch absorption as compared to the $\mathrm{C}=\mathrm{O}$ stretch signal confirming the loss of double bonds relative to carbonyl moieties (Fig. 3b).

To further verify that this residue is actually produced by HABI-initiated radical polymerization, we subjected PU-1 to swelling in a mixture of methyl acrylate (MA) and $50 \mathrm{~mol} \%$ MMP. After mechanical activation by freezing this allowed us to analyse the obtained sample by ${ }^{1} \mathrm{H}-\mathrm{NMR}$ spectroscopy. Fig. 3c clearly shows the vanishing of MA's characteristic acrylic protons (5.80-6.50 ppm) as well as a shift of MA's methylgroup (3.77 ppm). Concurrently, broad signals indicative of 

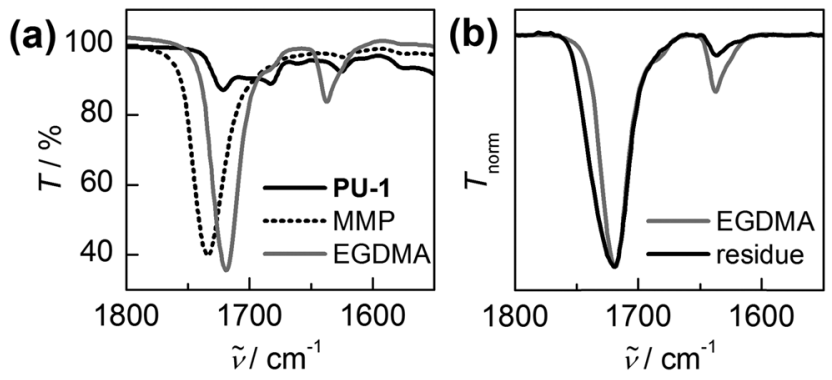

(c)

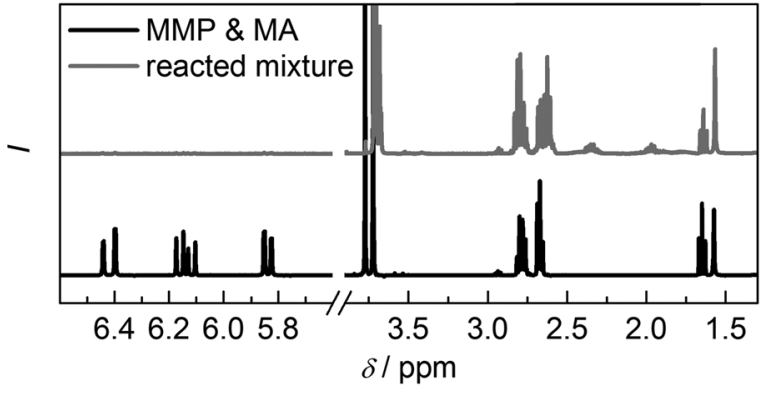

Fig. 3 FTIR transmission spectra of (a) PU-1, PU-1 swollen with MMP and PU-1 swollen with EGDMA as well as (b) PU-1 swollen in EGDMA and the insoluble residue obtained after mechanically induced polymerization normalized to the $\mathrm{C}=\mathrm{O}$ stretch signal at $1719 \mathrm{~cm}^{-1}$. (c) ${ }^{1} \mathrm{H}$-NMR spectra of a mixture of MA with $50 \mathrm{~mol} \%$ MMP before and after subjecting it to the mechanically induced oligomerization by freezing swollen PU-1.

PMA's alkylic backbone (1.8-2.5 ppm) appear. Together with the remaining triplet at $1.65 \mathrm{ppm}$, suggesting incomplete consumption of the thiol, and GPC measurements (Fig. S6, ESI $\dagger$ ) this unequivocally demonstrates a successful mechanically induced radical oligomerization.

In conclusion, employing the mechanoresponsive HABI moiety we showed here for the first time that mechanochemically generated radicals in polymeric architectures can be used for stress-sensing giving rise to coloured stable radicals as well as for the initiation of a secondary radical reaction. We believe that this proof of concept contributes considerably to the fundamental understanding of how we can alter the destructive nature of mechanical stress to perform constructive chemistry and anticipate that stress-induced crosslinking with the HABI mechanophore may be applied even more broadly when combined with non-volatile acrylate and thiol species.

Robert Göstl was supported by the Deutsche Forschungsgemeinschaft (DFG) through a research fellowship - GO 2634/1-1. This work was supported by the Dutch Ministry of Education, Culture and Science (Gravity program 024.001.035).

\section{References}

1 M. K. Beyer and H. Clausen-Schaumann, Chem. Rev., 2005, 105, 2921-2948.

2 M. M. Caruso, D. A. Davis, Q. Shen, S. A. Odom, N. R. Sottos, S. R. White and J. S. Moore, Chem. Rev., 2009, 109, 5755-5798.

3 Z. S. Kean and S. L. Craig, Polymer, 2012, 53, 1035-1048.

4 R. Groote, R. T. M. Jakobs and R. P. Sijbesma, Polym. Chem., 2013, 4, $4846-4859$

5 J. Li, C. Nagamani and J. S. Moore, Acc. Chem. Res., 2015, 48, 2181-2190.

6 D. R. T. Roberts and S. J. Holder, J. Mater. Chem., 2011, 21, 8256-8268.

7 F. Ciardelli, G. Ruggeri and A. Pucci, Chem. Soc. Rev., 2013, 42, 857-870.

8 R. T. M. Jakobs, S. Ma and R. P. Sijbesma, ACS Macro Lett., 2013, 2, 613-616.

9 R. T. M. Jakobs and R. P. Sijbesma, Organometallics, 2012, 31, 2476-2481.

10 A. Piermattei, S. Karthikeyan and R. P. Sijbesma, Nat. Chem., 2009, 1, 133-137.

11 H. Zhang, F. Gao, X. Cao, Y. Li, Y. Xu, W. Weng and R. Boulatov, Angew. Chem., Int. Ed., 2016, 55, 3040-3044.

12 J. Wang, I. Piskun and S. L. Craig, ACS Macro Lett., 2015, 4, 834-837.

13 A. L. B. Ramirez, Z. S. Kean, J. A. Orlicki, M. Champhekar, S. M. Elsakr, W. E. Krause and S. L. Craig, Nat. Chem., 2013, 5, 757-761.

14 A. L. Black, J. A. Orlicki and S. L. Craig, J. Mater. Chem., 2011, 21, $8460-8465$.

15 K. Imato, T. Kanehara, T. Ohishi, M. Nishihara, H. Yajima, M. Ito, A. Takahara and H. Otsuka, ACS Macro Lett., 2015, 4, 1307-1311.

16 K. Imato, A. Irie, T. Kosuge, T. Ohishi, M. Nishihara, A. Takahara and H. Otsuka, Angew. Chem., Int. Ed., 2015, 54, 6168-6172.

17 K. Imato, M. Nishihara, T. Kanehara, Y. Amamoto, A. Takahara and H. Otsuka, Angew. Chem., Int. Ed., 2012, 51, 1138-1142.

18 S. L. Malhotra, J. Macromol. Sci., Part A: Pure Appl.Chem., 1982, 18, 1055-1085.

19 C. Keqiang, S. Ye, L. Huilin and X. Xi, J. Macromol. Sci., Part A: Pure Appl.Chem., 1986, 23, 455-469.

20 H. Çatalgil-Giz and Y. Hepuzer, J. Appl. Polym. Sci., 2000, 77, 1950-1953.

21 M. Degirmenci, H. Catalgil-Giz and Y. Yagci, J. Polym. Sci., Part A: Polym. Chem., 2004, 42, 534-540.

22 T. Hayashi, K. Maeda, S. Shida and K. Nakada, J. Chem. Phys., 1960, 32, 1568.

23 T. Hayashi, K. Maeda and M. Morinaga, Bull. Chem. Soc. Jpn., 1964, 37, 1563-1564.

24 T. Hayashi and K. Maeda, Bull. Chem. Soc. Jpn., 1965, 38, 685-686. 25 D. M. White and J. Sonnenberg, J. Am. Chem. Soc., 1966, 88, 3825-3829. 26 B. M. Monroe and G. C. Weed, Chem. Rev., 1993, 93, 435-448.

27 T. Iwamura and S. Nakamura, Polymer, 2013, 54, 4161-4170.

28 T. Iwamura and M. Sakaguchi, Macromolecules, 2008, 41, 8995-8999.

29 D. Ahn, S. S. Sathe, B. H. Clarkson and T. F. Scott, Dent. Mater., 2015, 31, 1075-1089.

30 S. S. Sathe, D. Ahn and T. F. Scott, Ind. Eng. Chem. Res., 2015, 54, 4203-4212.

31 A. Anastasaki, C. Waldron, P. Wilson, C. Boyer, P. B. Zetterlund, M. R. Whittaker and D. Haddleton, ACS Macro Lett., 2013, 2, 896-900.

32 Y. Sakaino, H. Kakisawa, T. Kusumi and K. Maeda, J. Org. Chem., 1979, 44, 1241-1244.

33 Y. Chen and R. P. Sijbesma, Macromolecules, 2014, 47, 3797-3805.

34 M. Schaefer, B. Icli, C. Weder, M. Lattuada, A. F. M. Kilbinger and Y. C. Simon, Macromolecules, 2016, 49, 1630-1636.

35 P. A. May, N. F. Munaretto, M. B. Hamoy, M. J. Robb and J. S. Moore, ACS Macro Lett., 2016, 5, 177-180.

36 K. B. Abbas and R. S. Porter, J. Polym. Sci., Polym. Chem. Ed., 1976, 14, 553-564. 